\title{
A Quasi-Linear Kinetic Equation for Cosmic Rays in the Interplanetary Medium
}

\author{
J. G. LUhMANN ${ }^{1}$ \\ Department of Physics and Astronomy, University of Maryland, College Park, Maryland 20742
}

\begin{abstract}
A kinetic equation for interplanetary cosmic rays is set up with the aid of weak plasma turbulence theory for an idealized radially symmetric model of the interplanetary magnetic field. As a starting point this treatment invokes the Vlasov equation instead of the traditional Fokker-Planck equation. Quasilinear theory is applied to obtain a momentum diffusion equation for the heliocentric frame of reference which describes the interaction of cosmic rays with convecting magnetic irregularities in the solar wind plasma. Under restricted conditions the well-known equation of solar modulation can be obtained from this kinetic equation.
\end{abstract}

\section{INTRODUCTION}

Parker [1958, 1965] originally formulated the well-known transport equation of solar modulation

$$
\begin{aligned}
\frac{\partial U}{\partial t}+\frac{1}{r^{2}} \frac{\partial}{\partial r}\left(r^{2} u U\right)-\frac{1}{3 r^{2}} \frac{\partial}{\partial r} & \left(r^{2} u\right) \frac{\partial}{\partial T}(\alpha T U) \\
& -\frac{1}{r^{2}} \frac{\partial}{\partial r}\left(r^{2} \kappa \frac{\partial U}{\partial r}\right)=0
\end{aligned}
$$

This equation describes the cosmic ray density $U(r, T, t)$ at kinetic energy $T$ in a spherically symmetric space where elastic scattering centers, moving radially at the solar wind speed $u$, cause spatial diffusion and energy loss. The relationship between the spatial diffusion coefficient $\kappa$ and the magnetic fluctuations observed in the interplanetary medium [Coleman, 1966] was described explicitly by Jokipii [1966]. By considering a Fokker-Planck equation [Chandrasekhar, 1943] for cosmic rays in a frame of reference moving with the solar wind plasma, Jokipii, and later Hasselmann and Wibberenz [1968], derived the diffusion coefficient for the case of stationary magnetic turbulence and a homogeneous average magnetic field. Gleeson and Axford [1967] subsequently demonstrated that a Boltzmann equation for a radial geometry leads directly to the modulation equation (1) for the heliocentric frame of reference. However, their model invoked a collision term to describe the wave-particle interactions that occur in interplanetary space. As an alternative, this paper shows how the Vlasov equation and quasi-linear theory can be applied to solar modulation. The result is a kinetic equation that embodies both the geometrical aspects of the Gleeson and Axford model and the wave-particle interaction effects discussed by Jokipii [1966].

In the quasi-linear theory of weakly turbulent plasmas the distribution function and the electromagnetic fields are expressed as the sum of an averaged component and a perturbation. When these linearized quantities are substituted into the Vlasov equation, a plasma kinetic equation that describes diffusion in velocity space is obtained [Kennel and Engelmann, 1966]. Moreover, the diffusion tensor can be expressed in terms of the power spectrum of the turbulent field fluctuations. In this paper, cosmic rays are regarded as a small relativistic component of the interplanetary plasma that interacts

${ }^{1}$ Now at Space Physics Laboratory, The Aerospace Corporation, El Segundo, California 90245.

Copyright (C) 1976 by the American Geophysical Union. resonantly with the ambient field fluctuations. With this point of view, the quasi-linear result of Kennel and Engelmann is specialized to resonant interactions between particles and the convecting field inhomogeneities in the solar wind to obtain a kinetic equation describing interplanetary cosmic ray transport.

A quasi-linear diffusion equation has also been applied to cosmic ray transport in the interstellar medium [Kulsrud and Pearce, 1969]. In interstellar space the resonant interactions occur between the particles and Alfvén waves generated by the cosmic rays themselves [Lerche, 1967; Wentzel, 1968]. Under these circumstances the wave spectrum and particle distribution are interdependent, and their evolution must be considered together. However, in interplanetary space the field fluctuations are evidently quasi-static magnetic structures and waves in the solar wind generated near its source or hydromagnetic waves produced by instabilities of the expanding solar wind plasma [Coleman, 1968; Scarf, 1970]. This turbulence can be considered as a part of the ambient field environment in which the cosmic rays move, and a fully self-consistent treatment relating the waves to the distribution of cosmic rays is not required.

The limitations on the applicability of quasi-linear theory to cosmic ray transport have been discussed in detail elsewhere [Klimas and Sandri, 1973; Kaiser et al., 1973; Goldstein, 1975]. It has been demonstrated [Kaiser, 1975] by means of numerical simulations that quasi-linear theory does not accurately describe charged particle transport for pitch angles near $90^{\circ}$ in moderately turbulent magnetic field models of limited dimensionality. On the other hand, in some applications, quasilinear theory has been shown to approximate the results of more exact analyses of particle interactions with turbulent fields with relative mathematical simplicity [Krall et al., 1972; Jokipii and Lerche, 1973]. However, the present discussion is not intended to justify the application of quasi-linear theory in this case but rather to describe an alternative viewpoint from which it can be applied to the modulation problem.

In the following discussion it is assumed that the average interplanetary magnetic field has a radial geometry. The interplanetary field turbulence is modeled by transverse random magnetic fluctuations which are distributed axisymmetrically about the average field direction. Because the waves observed in the solar wind plasma generally have phase velocities about an order of magnitude less than the solar wind speed $u \sim 400$ $\mathrm{km} / \mathrm{s}$, it is also assumed that these fluctuations have zero phase velocity in the plasma frame and move radially with respect to 
the sun at the plasma bulk speed. In the context of this model the cosmic rays interact with 'waves' of phase velocity $\boldsymbol{u}$ that result from the convection of the solar wind.

Following a brief discussion of its derivation the quasilinear kinetic equation is restated below in terms of the interplanetary fields and the resonant cosmic ray distribution. Finally, the relationship between this plasma kinetic equation for the interplanetary cosmic ray distribution and the well-known modulation equation [Parker, 1965; Gleeson and Axford, 1967] is discussed. It is demonstrated that the modulation equation is obtained only under restricted conditions and consequently excludes some features of the quasi-linear equation for interplanetary transport.

\section{Quasi-Linear Kinetic Equation}

The Vlasov equation or collisionless Boltzmann equation

$$
\frac{\partial F}{\partial t}+\nabla \cdot \nabla_{s} F+Z e\left(\mathrm{E}+\frac{1}{c} \nabla \times \mathrm{B}\right) \cdot \nabla_{\mathrm{p}} F=0
$$

describes the evolution of a particle distribution function $F($ s, $\mathrm{p}, t)$ under the influence of electromagnetic forces in the $a b-$ sence of collisions. In (2), $t$ is time, $v$ is particle velocity, $p$ is momentum, $Z e$ is charge, $s$ is a spatial vector, $c$ is the speed of light, and $E$ and $B$ are the electric and magnetic fields.

Quasi-linear theory separates the fields into two components

$$
\begin{aligned}
& \mathbf{E}=\langle\mathbf{E}\rangle+\mathbf{8 E} \\
& \mathbf{B}=\langle\mathbf{B}\rangle+\mathbf{8 B}
\end{aligned}
$$

where angle brackets denote the ensemble averaged values of the fields [Batchelor, 1946] and the perturbations $\mathbf{6 E}$ and $\mathbf{6 B}$ represent rapidly fluctuating fields whose average values are zero. The distribution function is similarly expressed as a perturbed quantity:

$$
F=f+\delta f
$$

where the slowly evolving average distribution $f=\langle\boldsymbol{F}\rangle$ is the component of interest.

If the average electric field $\langle\mathrm{E}\rangle$ is set equal to zero, if particle density gradients are restricted to directions parallel to an average magnetic field $\langle\mathbf{B}\rangle$ which has negligible curvature, and if $f$ is axisymmetric with respect to the direction of $\langle\mathbf{B}\rangle$, the substitution of the above expansions into the Vlasov equation leads to the kinetic equation

$$
\begin{aligned}
\frac{\partial \bar{f}}{\partial t}+v_{\|} \frac{\partial \bar{f}}{\partial s_{\|}}-\frac{1}{2} & \frac{p_{\perp}}{\langle B\rangle} \frac{\partial\langle B\rangle}{\partial s_{\|}} \\
& \cdot\left(v_{\perp} \frac{\partial}{\partial p_{\|}}-v_{\|} \frac{\partial}{\partial p_{\perp}}\right) \bar{f}=\frac{\partial}{\partial \bar{p}} \cdot D \cdot \frac{\partial \bar{f}}{\partial \bar{p}}
\end{aligned}
$$

In (5) the bar over $f$ indicates that it has been averaged over gyrophase. Vector components parallel and perpendicular to the average magnetic field direction are designated by the parallel and perpendicular subscripts, respectively. The lefthand side of (5) describes particle conservation in a magnetic field $(B)$. The velocity space diffusion operator on the righthand side, which contains the diffusion tensor $D$ that depends on the power spectrum of the field turbulence, has been discussed by Kennel and Engelmann [1966], Hall and Sturrock [1967], $W u$ [1968], and others. This collisionlike term describes the effects of wave-particle interactions on $\bar{f}$. Here, it will be appropriate to concentrate on the form of the diffusion term for the specific case where the field turbulence consists of electromagnetic waves which propagate parallel to the direction of the average magnetic field $\langle\mathbf{B}\rangle$ and where $\bar{f}$ represents a distribution of resonant particles. Under these circumstances the right-hand side of (5) can be written in the form

$$
\begin{aligned}
& \frac{\partial}{\partial \bar{p}} \cdot D \cdot \frac{\partial \bar{f}}{\partial \bar{p}}=2\left(\frac{\pi Z e}{c}\right)^{2} \int_{0}^{\infty} d k_{\|} \varepsilon_{B}\left(k_{\|}\right) \\
& \cdot\left[v_{\perp} \frac{\partial}{\partial p_{\|}}+\left(\frac{\omega}{k_{\|}}-v_{\|}\right) \frac{1}{p_{\perp}} \frac{\partial}{\partial p_{\perp}} p_{\perp}\right] \delta\left(\omega-k_{\|} v_{\|} \pm \Omega\right) \\
& \cdot\left[v_{\perp} \frac{\partial}{\partial p_{\|}}+\left(\frac{\omega}{k_{\|}}-v_{\|}\right) \frac{\partial}{\partial p_{\perp}}\right] \bar{f}
\end{aligned}
$$

In $(6), \omega=\omega\left(k_{\|}\right)$is the wave dispersion relation which depends on $k_{11}$, the wave number of the field fluctuations; the spectral function $\varepsilon_{B}\left(k_{\| \mid}\right)$describes the distribution of magnetic energy $\langle\delta \mathrm{B} \cdot \delta \mathrm{B}\rangle / 8 \pi$ in the waves; and the cyclotron frequency $\Omega$ is equal to $Z e\langle B\rangle / \gamma m c$, where $m$ is the rest mass and $\gamma$ is the Lorentz factor $\left(1-v^{2} / c^{2}\right)^{-1 / 2}$. The Dirac delta function $\delta\left(\omega-k_{\|} v_{\|} \pm \Omega\right)$ appears because (6) describes a resonant process. It serves to isolate the spectral power at the resonant wave number $k_{\|}{ }^{0}$ which is defined by the resonance condition

$$
\omega-k_{\|}{ }^{0} v_{\|} \pm \Omega=0
$$

Equation (7) expresses the idea that resonance occurs when the Doppler-shifted wave frequency seen by the particle is equal to its gyrofrequency. So far, no restrictions have been imposed on the waves that enter into (6) except that they are electromagnetic and propagate parallel to the average magnetic fieid. In fact, the diffusion operator (6) is essentially identical to the form given by Davidson [1972, chapter 12] for electron resonant interactions with whistler noise.

\section{Application to Solar Modulation}

In the environment of interplanetary space it is convenient to regard the turbulent field fluctuations as electromagnetic waves obeying the dispersion relation

$$
\omega=k_{\|} u
$$

These 'waves,' which propagate radially in the direction of $\langle\mathbf{B}\rangle$, are produced by the convection of magnetic inhomogeneities. The Fourier expansion describing the turbulence is one-dimensional because in this idealized model, each field line effectively has its own spectrum. The transverse magnetic components of these waves $\mathbf{\delta} \mathbf{B}$ are the fluctuations observed from spacecraft. The electric component given by

$$
\delta \mathrm{E}=-\mathrm{u} \times \delta \mathrm{B} / \mathrm{c}
$$

is produced by the convection of the magnetic fluctuations with respect to the fixed frame. In the previous Fokker-Planck treatments of solar modulation [Jokipii, 1966] a linearized representation of the magnetic field was also invoked, but the electric field was neglected because the calculations were carried out in the solar wind frame where $u$ is zero.

With the substitution of the specialized dispersion relation (8) into (6), (5) and (6) provide a kinetic equation for interplanetary cosmic rays. A particularly convenient form is obtained if the variables $p$, the magnitude of the momentum, and $\mu=p_{\|} / p$, the cosine of the pitch angle, are adopted. The resulting kinetic equation is given by

$$
\begin{gathered}
\frac{\partial \bar{f}}{\partial t}+\mu v \frac{\partial \bar{f}}{\partial r}+\frac{v}{r}\left(1-\mu^{2}\right) \frac{\partial \bar{f}}{\partial \mu}=\frac{\partial}{\partial \mu}\left(D_{\mu \mu} \frac{\partial \bar{f}}{\partial \mu}+D_{\mu p} \frac{\partial \bar{f}}{\partial p}\right) \\
+\frac{1}{p} \frac{\partial}{\partial p} p^{2}\left(D_{p \mu} \frac{\partial \bar{f}}{\partial \mu}+D_{p p} \frac{\partial \bar{f}}{\partial p}\right)
\end{gathered}
$$




$$
\begin{gathered}
D_{\mu \mu}=\left(\frac{2 \pi Z e}{c}\right)^{2} \frac{v^{2}}{p^{2}} \frac{\mathcal{E}_{B}\left(k_{\|}{ }^{0}\right)}{(1-u / \mu v)} \frac{\left(1-\mu^{2}\right)}{|\mu|}(1 \quad \mu u / v)^{2} \\
D_{p \mu}=\left(\frac{2 \pi Z e}{c}\right)^{2} \frac{v^{2}}{p^{2}} \frac{\mathcal{E}_{B}\left(k_{\|}{ }^{0}\right)}{(1-u / \mu v)} \frac{(1}{|\mu|} \mu^{\left.\mu^{2}\right)} \\
\cdot(1-\mu u / v) p u / v \\
D_{p p}=\left(\frac{2 \pi Z e}{c}\right)^{2} \frac{v^{2}}{p^{2}} \frac{\mathcal{E}_{B}\left(k_{\|}{ }^{0}\right)}{(1-u / \mu v)} \frac{\left(1-\mu^{2}\right)}{|\mu|}(p u / v)^{2}
\end{gathered}
$$

and where $k_{\| 1}{ }^{0}=|\Omega| /|u-\mu v|$ is the resonant wave number for cosmic rays with parallel velocity $\mu v$ and cyclotron frequency $\Omega$. The first two terms on the left-hand side of $(10)$ are selfevident. The third term is a consequence of the radial geometry of the average magnetic field. As discussed by Roelof [1969], this term is related to the adiabatic invariance of the magnetic moment of the particle. The right-hand side of $(10)$ describes the momentum diffusion produced by the interaction of cosmic rays with the interplanetary field fluctuations. This general form of the diffusion operator was discussed by Melrose [1969] in connection with particle interactions with Alfvén waves. The terms that are proportional to powers of $u / v$ represent an energy exchange between the particles and the turbulence. These are related to the fluctuating electric field $\mathbf{\delta E}$ which is produced by the convection of the magnetic fluctuations. The remaining terms are related to pitch angle diffusion. In Appendix A the kinetic equation (10) is translated into a quasi Fokker-Planck form for comparison with the equation considered by Jokipii [1966].

\section{Discussion}

The well-known modulation equation (1) [Parker, 1965], expressed in terms of the phase space density $f_{0}(r, p, t)$ [Gleeson and Axford, 1967; Fisk et al., 1973], is given by

$$
\begin{aligned}
\frac{\partial f_{0}}{\partial t}+u \frac{\partial f_{0}}{\partial r}-\frac{1}{r^{2}} \frac{\partial}{\partial r}\left(r^{2} \kappa\right. & \left.\frac{\partial f_{0}}{\partial r}\right) \\
& -\frac{1}{3 r^{2}} \frac{\partial}{\partial r}\left(r^{2} u\right) p \frac{\partial f_{0}}{\partial p}=0
\end{aligned}
$$

The phase space density $f_{0}$ is related to $\bar{f}$ by integration over pitch angle:

$$
f_{0}=\int^{1} \bar{f} d \mu
$$

The spatial diffusion coefficient $\kappa$ in (11) can be expressed in terms of the pitch angle diffusion coefficient $D_{\mu \mu}$ as

$$
\kappa=v^{2} \int_{0}^{1} \frac{\mu d \mu}{2} \int_{0}^{\mu} \frac{\left(1-\mu^{\prime 2}\right)}{D_{\mu^{\prime} \mu^{\prime}}} d \mu^{\prime}
$$

[Jokipii, 1966, 1968; Hasselmann and Wibberenz, 1970] when the power spectrum of magnetic fluctuations is not too steep. This relationship between the spatial and pitch angle diffusion coefficients can be derived from (10), in conjunction with the modulation equation (11), if certain constraints are imposed on the parallel velocity $\mu v$ and the form of the distribution function $\bar{f}$.

At typical cosmic ray energies $(\sim 1 \mathrm{GeV})$ the solar wind velocity $u$ is small in comparison with the parallel component of the particle velocity $\mu v$ if the pitch angle is not close to $90^{\circ}$. In this case $(u \ll \mu v)$ the approximations $(1-u / v) \approx 1$ and $(1$ $-\mu u / v) \approx 1$ can be made. With these two substitutions, (10) can be simplified to

$$
\begin{gathered}
\frac{\partial \bar{f}}{\partial t}+\mu v \frac{\partial \bar{f}}{\partial r}+\frac{v}{r} \\
\left(1-\mu^{2}\right) \frac{\partial \bar{f}}{\partial \mu}=\frac{\partial}{\partial \mu} D_{\mu \mu}\left(\frac{\partial \bar{f}}{\partial \mu}+\frac{u}{v} p \frac{\partial \bar{f}}{\partial p}\right) \\
+\frac{1}{p^{2}} \frac{\partial}{\partial p} p^{3} \frac{u}{v} D_{\mu \mu}\left(\frac{\partial \bar{f}}{\partial \mu}+\frac{u}{v} p \frac{\partial \bar{f}}{\partial p}\right)
\end{gathered}
$$

and the pitch angle diffusion coefficient $D_{\mu \mu}$ takes the form obtained by Jokipii [1966]:

$$
D_{\mu \mu} \cong\left(\frac{2 \pi Z e}{c}\right)^{2} \frac{v^{2}}{p^{2}} \varepsilon_{B}\left(k_{\|}{ }^{0}\right) \frac{\left(1-\mu^{2}\right)}{|\mu|}
$$

In the diffusion regime [Earl, 1974], where the magnetic power spectrum $\varepsilon_{B} \propto k^{-q}$ has an index $q$ that is less than 2 , the distribution function can be expressed as the sum of $f_{0}$ and an anisotropy $f_{1}(\mu)$, which is an odd function of $\mu$ :

$$
\bar{f}=f_{0}+f_{1}(\mu)
$$

With the exception of $f_{0}$ the even terms in the series expansion of $\bar{f}$ are neglected. The anisotropy $f_{1}$ represents the effect of a net parallel velocity of the particles.

When the expansion (16) is substituted for $\bar{f},(14)$ can be separated into two equations in $f_{0}$ and $f_{1}$ which equate the terms that are even in $\mu$,

$$
\begin{aligned}
\frac{\partial f_{0}}{\partial t}+\mu v \frac{\partial f_{1}}{\partial r}+ & \frac{v}{r}\left(1-\mu^{2}\right) \frac{\partial f_{1}}{\partial \mu}=\frac{\partial}{\partial \mu} D_{\mu \mu}\left(\frac{u}{v} p \frac{\partial f_{1}}{\partial p}\right) \\
& +\frac{1}{p^{2}} \frac{\partial}{\partial p} p^{3} \frac{u}{v} D_{\mu \mu}\left(\frac{\partial f_{1}}{\partial \mu}+\frac{u}{v} p \frac{\partial f_{0}}{\partial p}\right)
\end{aligned}
$$

and odd in $\mu$

$$
\begin{aligned}
\frac{\partial f_{1}}{\partial t}+\mu v \frac{\partial f_{0}}{\partial r}=\frac{\partial}{\partial \mu} D_{\mu \mu}\left(\frac{\partial f_{1}}{\partial \mu}\right. & \left.+\frac{u}{v} p \frac{\partial f_{0}}{\partial p}\right) \\
& +\frac{1}{p^{2}} \frac{\partial}{\partial p} p^{4} \frac{u^{2}}{v^{2}} D_{\mu \mu} \frac{1}{\partial p}
\end{aligned}
$$

If $\partial f_{1} / \partial t$ and terms proportional to $(u / v)^{2}$ are neglected, (18) can be solved for $f_{1}$ :

$$
f_{1}(\mu)=-\frac{v}{2} \frac{\partial f_{0}}{\partial r} \int \frac{\left(1-\mu^{2}\right)}{D_{\mu \mu}} d \mu-\frac{u}{v} p \frac{\partial f_{0}}{\partial p} \mu
$$

For the restricted case under consideration the anisotropy $f_{1}$ is made up of two components. The first is related to pitch angle diffusion and the gradient of $f_{0}$. This term provides the parallel spatial diffusion that is characterized by the coefficient $\kappa$ in (11). Evidently, pitch angle diffusion can produce spatial effects in a plasma if a density gradient $\partial f_{0} / \partial r$ is present. A steady density gradient is maintained in the interplanetary cosmic ray distribution because the solar wind plasma has a transit time $r / u$ comparable to the characteristic cosmic ray diffusion time from interstellar space to $r$. The second contribution to $f_{1}$ is from the relative motion of the turbulent fields at $u$ and is related to the Compton-Getting effect [Compton and Getting, 1935]. The substitution of (19) into (17) leads to an equation for $f_{0}$ that is identical to (11) if terms proportional to $(u / v)^{2}$ are again neglected. Hence in retrospect it is seen that (10) reduces to the modulation equation (11) when the following assumptions are made: (1) the magnetic spectral index is less than 2 , (2) $u \ll \mu v$, (3) $\bar{f}=f_{0}+f_{1}$, (4) $\partial f_{1} / \partial t=0$, and (5) $(u / v)^{2}=0$. Clearly, if effects outside of the framework of these assumptions are under consideration, the more general (10) must be invoked. 


\section{SUMMARY}

It has been demonstrated that quasi-linear theory and the Vlasov equation can be used to obtain a kinetic equation for cosmic rays in interplanetary space. The kinetic equation embodies both the radial expansion of the interplanetary medium and the resonant interactions between the cosmic rays and the interplanetary magnetic field fluctuations. The only limitations on the validity of this kinetic equation, within the framework of the idealized model of the interplanetary medium used here, are the limitations inherent in the use of perturbation expansions in turbulence problems and in their use in nonuniform plasmas. The plasma physical approach to cosmic ray modulation that has been taken here is potentially useful for assessing the effects of interplanetary medium asymmetries on interplanetary transport. In particular, the spiral magnetic field configuration that is the result of the solar rotation should eventually be included in modulation theory, in addition to the variation of the solar wind properties with heliocentric distance and heliolatitude. Also, the transport of the solar and Jovian components of the interplanetary cosmic radiation can be examined from a similar point of view.

\section{APPENdix A: Fokker-Planck Equation FOR SOLAR MOdULATION}

The right-hand side of the kinetic equation (10) can be cast in the characteristic Fokker-Planck form [Chandrasekhar, 1943]

$$
\begin{aligned}
\frac{\partial \bar{f}}{\partial t} & +\mu v \frac{\partial \ddot{f}}{\partial r}+\frac{v}{r}\left(1-\mu^{2}\right) \frac{\partial \bar{f}}{\partial \mu}=\frac{1}{2} \frac{\partial^{2}}{\partial \mu^{2}}\left[\frac{\left\langle(\Delta \mu)^{2}\right\rangle}{\Delta t} \bar{f}\right] \\
& -\frac{\partial}{\partial \mu}\left(\frac{\langle\Delta \mu\rangle}{\Delta t} \bar{f}\right)+\frac{1}{p^{2}} \frac{\partial}{\partial \mu} p^{2}\left(\frac{\langle\Delta \mu \Delta p\rangle}{\Delta t} \bar{f}\right) \\
& +\frac{1}{2 p^{2}} \frac{\partial^{2}}{\partial p^{2}} p^{2}\left[\frac{\left\langle(\Delta p)^{2}\right\rangle}{\Delta t} \bar{f}\right]-\frac{1}{p^{2}} \frac{\partial}{\partial p} p^{2}\left(\frac{\langle\Delta p\rangle}{\Delta t} \bar{f}\right)
\end{aligned}
$$

if the Fokker-Planck coefficients are given by

$$
\begin{aligned}
\left\langle(\Delta \mu)^{2}\right\rangle / \Delta t & =2 D_{\mu \mu} \\
\left\langle(\Delta p)^{2}\right\rangle / \Delta t & =2 D_{p p} \\
\langle\Delta \mu \Delta p\rangle / \Delta t & =2 D_{\mu p} \\
\langle\Delta \mu\rangle / \Delta t & =\frac{\partial}{\partial \mu} D_{\mu \mu}+\frac{1}{p^{2}} \frac{\partial}{\partial p} p^{2} D_{\mu p} \\
\langle\Delta p\rangle / \Delta t & =\frac{\partial}{\partial \mu} D_{\mu p}+\frac{1}{p^{2}} \frac{\partial}{\partial p} p^{2} D_{p p}
\end{aligned}
$$

Jokipii [1966] invoked the Fokker-Planck equation

$$
\begin{aligned}
& \frac{\partial \bar{f}^{\prime}}{\partial t}+\mu^{\prime} v^{\prime} \frac{\partial \bar{f}^{\prime}}{\partial r}=\frac{1}{2} \frac{\partial}{\partial \mu^{\prime}}\left[\frac{\left\langle\left(\Delta \mu^{\prime}\right)^{2}\right\rangle}{\Delta t} \bar{f}^{\prime}\right] \\
&-\frac{\partial}{\partial \mu^{\prime}}\left[\frac{\left\langle\Delta \mu^{\prime}\right\rangle}{\Delta t} \bar{f}^{\prime}\right]=\frac{1}{2} \frac{\partial}{\partial \mu^{\prime}} \frac{\left\langle\left(\Delta \mu^{\prime}\right)^{2}\right\rangle}{\Delta t} \frac{\partial f^{\prime}}{\partial \mu^{\prime}}
\end{aligned}
$$

where the primes are added to emphasize that this equation applies to the solar wind frame of reference.

Pitch angle diffusion, described by a pitch angle diffusion coefficient $\frac{1}{2}\left((\Delta \mu)^{2}\right) / \Delta t$, appears in both cases. However, (A1) also includes the additional Fokker-Planck coefficients involving the momentum $p$ and the term $(v / r)\left(1-\mu^{2}\right) \partial f / \partial \mu$ that is related to the divergence of the magnetic field. These additional terms provide the so-called adiabatic deceleration term $-(1 / 3 r)(\partial / \partial r)\left(r^{2} u\right) p \partial f_{0} / \partial p$ in the modulation equation
(11). Hence in the context of the model that has been considered in this paper, adiabatic deceleration can be attributed to the combination of a diverging average magnetic field and the fluctuating electric field (equation (9)) produced by the convecting magnetic irregularities.

Acknowledgments. The work described in this paper was included in a thesis submitted to the Astronomy Program of the Department of Physics and Astronomy, University of Maryland, in partial fulfillment of the requirements for the Ph.D. The thesis was completed under the guidance of James A. Earl. The author wishes to acknowledge helpful discussions with R. C. Davidson and D. G. Wentzel and to thank Michael Schulz of The Aerospace Corporation for his comments on the manuscript. This research was supported in part by an NDEA Fellowship and by NASA grants NGR 21-002-033 and NGR 21-002-066 while the author was at the University of Maryland. Current support is provided by Air Force contract F04701-75-C-0076.

The Editor thanks M. L. Goldstein and another referee for their assistance in evaluating this paper.

\section{REFERENCES}

Batchelor, G. K., The theory of axisymmetric turbulence, Proc. Roy. Soc, Ser. A, 186, 480, 1946.

Chandrasekhar, S., Stochastic problems in physics and astronomy, Rev. Mod. Phys., 15, 1, 1943.

Coleman, P. J., Jr., Variations in the interplanetary magnetic field: Mariner 2, 1, Observed properties, J. Geophys. Res., 71, 5509, 1966.

Coleman, P. J., Jr., Turbulence, viscosity, and dissipation in the solar-wind plasma, Astrophys. J., 153, 371, 1968.

Compton, A. H., and 1. A. Getting, An apparent effect of galactic rotation on the intensity of cosmic rays, Phys. Rev., 47, 817, 1935.

Davidson, R. C., Methods in Nonlinear Plasma Theory, Academic, New York, 1972.

Earl, J. A., The diffusive idealization of charged-particle transport in random magnetic fields, Astrophys. J., 193, 231, 1974.

Fisk, L. A., M. A. Forman, and W. I. Axford, Solar modulation of galactic cosmic rays, 3, Implications of the Compton-Getting coefficient, J. Geophys. Res., 78, 995, 1973.

Gleeson, L., and W. I. Axford, Cosmic rays in the interplanetary medium, Astrophys. J., 149, L115, 1967.

Goldstein, M. L., A nonlinear theory of cosmic ray pitch angle diffusion in homogeneous magnetostatic turbulence, NASA Doc. X-69275-64, 1975.

Hall, D. E., and P. A. Sturrock, Diffusion, scattering, and acceleration of particles by stochastic electromagnetic fields, Phys. Fluids, 10 , 2620,1967

Hasselmann, K., and G. Wibberenz, Scattering of charged particles by random electromagnetic fields, $Z$. Geophys., 34, 353, 1968.

Hasselmann, K., and G. Wibberenz, A note on the parallel diffusion coefficient, Astrophys. J., 162, 1049, 1970.

Jokipii, J. R., Cosmic ray propagation, 1, Charged particles in a random magnetic field, Astrophys. J., 146, 480, 1966.

Jokipii, J. R., Addendum and erratum to 'Cosmic ray propagation I,' Astrophys. J., 152, 671, 1968.

Jokipii, J. R., and I. Lerche, On the transport of charged particles in turbulent fields: Comparison of an exact solution with the quasilinear approximation, Plasma Phys., 15, 619, 1973.

Kaiser, T. B., Simulation of the velocity diffusion of charged particles in turbulent magnetic fields, Proc. Int. Conf. Cosmic Rays 14th, 861, 1975.

Kaiser, T. B., F. C. Jones, and T. J. Birmingham, Cosmic rays in a random magnetic field: Breakdown of the quasilinear derivation of the kinetic equation, Astrophys. J., 180, 239, 1973.

Kennel, C. F., and F. Engelmann, Velocity space diffusion from weak plasma turbulence in a magnetic field, Phys. Fluids. 9, 2377, 1966.

Klimas, A., and G. Sandri, The parallel diffusion of cosmic rays in a random magnetic field, Astrophys. J., 184, 955, 1973.

Krall, N. A., R. C. Davidson, and A. W. Trivelpiece, Space-averaged quasilinear theory and computer simulation experiments, Comments Plasma Phys. Contr. Fusion, I, 63, 1972.

Kulsrud, R., and W. P. Pearce, The effect of wave-particle interactions on the propagation of cosmic rays, Astrophys. J., 156, 445, 1969.

Lerche, I., Unstable magnetosonic waves in a relativistic plasma, Astrophys. J., 147, 689, 1967. 
Melrose, D. B., Resonant interaction of hydromagnetic waves with charged particles, Astrophys. Space Sci., 4, 143, 1969.

Parker, E. N., Cosmic ray modulation by the solar wind, Phys. Rev., 1.10, 1445, 1958.

Parker, E. N., The passage of energetic charged particles through interplanetary space, Planet. Space Sci., 13, 9, 1965.

Roelof, E. C., Propagation of solar cosmic rays in the interplanetary magnetic field, Lectures in High Energy Astrophysics, NASA Spec. Publ. 199, 1969.
Scarf, F. L., Microscopic structure of the solar wind, Space Sci. Rev., $11,234,1970$

Wentzel, D. G., Hydromagnetic waves excited by slowly streaming cosmic rays, Astrophys. J., 152, 987, 1968.

Wu, C. S., Unified theory of weakly turbulent plasmas, Phys. Fluids, $11,1733,1968$.

(Received September 9, 1975;

accepted January 28, 1976.) 